$47 / 25575(2)$

PREPARED FOR THE U.S. DEPARTMENT OF ENERGY, UNDER CONTRACT DE-AC02-76-CHO-3073

\title{
SINGULAR SURFACES IN THE OPEN FIELD LINE REGION OF A DIVERTED TOKAMAK
}

BY

A. REIMAN

PPPL-3099

PPPL-3099

UC-427

\author{
MAY 1995
}

$\int[5]\left[\begin{array}{l}D \\ \text { PAINCETON } \\ \text { PAAsMA PHYSICS }\end{array}\right.$

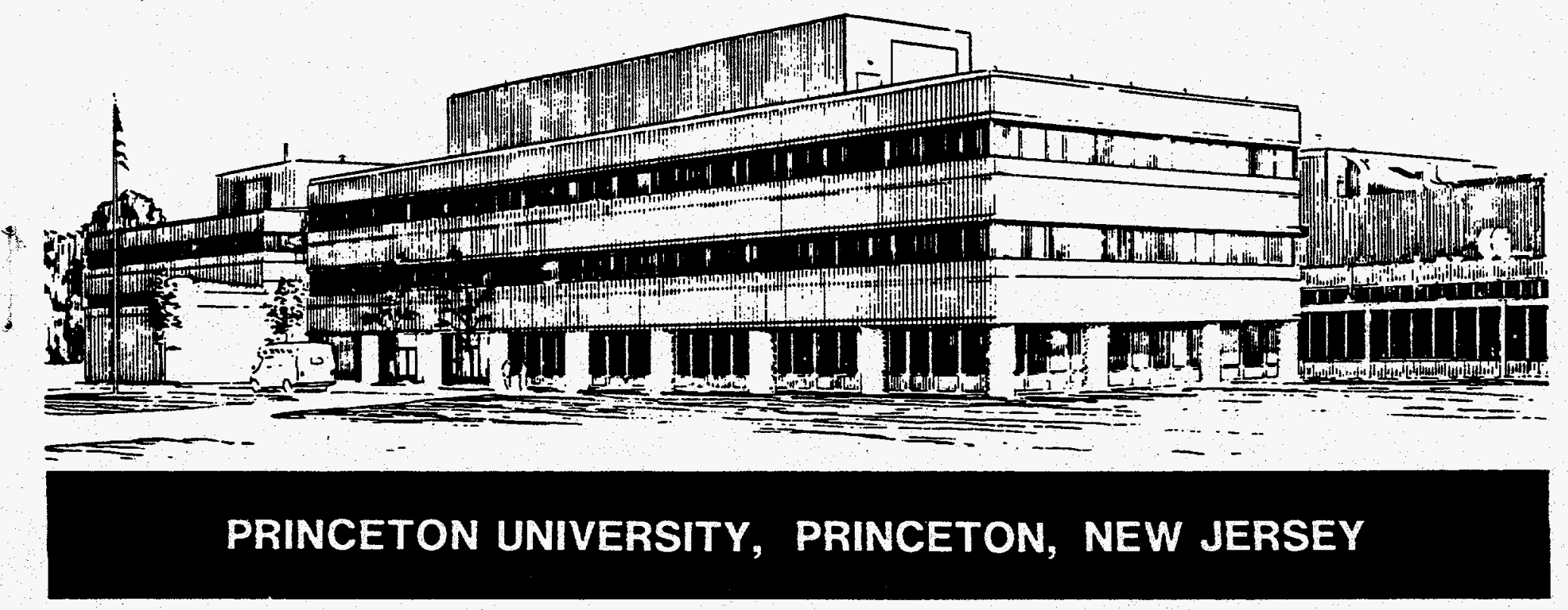




\title{
NOTICE
}

This report was prepared as an account of work sponsored by an agency of the United States Government. Neither the United States Government nor any agency thereof, nor any of their employees, makes any warranty, express or implied, or assumes any legal liability or responsibility for the accuracy, completeness, or usefulness of any information, apparatus, product, or process disclosed, or represents that its use would not infringe privately owned rights. Reference herein to any specific commercial produce, process, or service by trade name, trademark, manufacturer, or otherwise, does not necessarily constitute or imply its endorsement, recommendation, or favoring by the United States Government or any agency thereof. The views and opinions of authors expressed herein do not necessarily state or reflect those of the United States Government or any agency thereof.

\author{
NOTICE \\ This report has been reproduced from the best available copy. \\ Available in paper copy and microfiche. \\ Number of pages in this report: 34 \\ DOE and DOE contractors can obtain copies of this report from: \\ Office of Scientific and Technical Information \\ P.O. Box 62 \\ Oak Ridge, TN 37831 ; \\ (615) $576-8401$.
}

This report is publicly available from the:
National Technical Information Service
Department of Commerce
5285 Port Royal Road
Springfield, Virginia 22161
(703) $487-4650$




\section{DISCLAIMER}

Portions of this document may be illegible in electronic image products. Images are produced from the best available original document. 
April 3, 1995

\title{
Singular Surfaces in the Open Field Line Region of a Diverted Tokamak
}

\author{
A. Reiman \\ Plasma Physics Laboratory \\ James Forrestal Campus, Princeton University \\ P.O. Box 451 \\ Princeton, N. J. 08543
}

\begin{abstract}
The structure of the open field lines of a slightly nonaxisymmetric, poloidally diverted tokamak is explored by numerical integration of the field line equations for a simple model field. In practice, the nonaxisymmetry could be produced self-consistently by the nonlinear evolution of a free-boundary MHD mode, or it could be produced by field errors, or it could be imposed externally by design. In the presence of a nonaxisymmetric perturbation, the tokamak is shown to develop open field line regions of differing topology separated by singular surfaces. It is argued that the singular surfaces can be expected to play a role analogous to that of rational toroidal flux surfaces, in terms of constraining ideal MHD perturbations and thus constraining the free-energy that can be tapped by ideal MHD instabilities. The possibility of active control of free-boundary instabilities by means of currents driven on the open singular surfaces, which are directly accessible from the divertor plates, is discussed. Also discussed is the possibility of early detection of imminent disruptions through localized measurement of the singular surface currents.
\end{abstract}<smiles></smiles> 


\section{Introduction}

Rational surfaces and separatrices play a key role in constraining the ideal MHD dynamics of a toroidal plasma. In this paper we identify a set of singular surfaces in the open field line region of three-dimensional divertors which, we argue, play a role analogous to that which rational surfaces and separatrices play in a toroidal plasma. This is relevant for poloidal divertors in present day tokamaks, where field errors are typically of sufficient magnitude to introduce significant nonaxisymmetry in the neighborhood of the divertor separatrix. ${ }^{1}$ The presence of a nonaxisymmetric free-boundary MHD instability will itself introduce a perturbation of the divertor separatrix, so that, even if the tokamak is initially close to axisymmetry, the self-consistent nonlinear evolution of the mode may be influenced by the presence of singular surfaces in the open field-line region. One could also envision adding a nonaxisymmetric perturbation to a tokamak divertor for the purpose of influencing MHD modes through the constraints introduced by the singular surfaces. Stellarator divertors are intrinsically nonaxisymmetric, so that open singular surfaces also play a role there. The particular model we study in this paper is roore directly relevant to a perturbed tokamak poloidal divertor.

It is helpful to consider the analogy with rational toroidal flux surfaces. Those flux surfaces on which the safety factor, $q$, is a rational are called rational surfaces. Field lines on a rational toroidal flux surface close on themselves, so that they are topologically distinct from the surrounding field lines, which ergodically cover flux surfaces. Magnetic field perturbations at a rational surface which follow the pitch of the field lines (resonant perturbations) cause reconnection. The impossibility of reconnection within ideal MHD constrains the allowed magnetic field perturbations at the rational surface, and leads to the formation of surface currents to shield out perturbations that follow the pitch of the closed field lines. We will see in this paper that open field lines in a three-dimensional divertor have regions of differing topology, with singular surfaces separating the regions. We will argue that on an ideal MHD time scale the perturbations of the magnetic field at the singular surface are constrained by the requirement that the field lines cannot reconnect. We will also discuss an alternative way of looking at this, which involves an analysis of the singularities associated with the magnetic differential equation for the electrostatic potential along the field lines. ${ }^{2-4}$ This analysis, too identifies the 
open singular surfaces as the locus of three-dimensional reconnection.

The constraints on the allowable ideal MHD perturbations at singular surfaces reduces the free-energy available to drive instabilities. It also gives rise to surface currents intersecting the divertor plates. As we will discuss, the direct access suggests the possibility of external control over these currents, which would provide some measure of control over free-boundary MHD instabilities. It also suggests the possibility of early detection of imminent disruptions through localized measurement of the singular surface currents.

Figure 1 shows the conventional tokamak geometry in the absence of a divertor. There is a nominal edge outside of which the equilibrium current vanishes. In the figure, dashed lines indicate flux surfaces inside the edge, and solid lines indicate flux surfaces outside the edge. The ideal MHD stability properties of the tokamak are strongly affected by the presence or absence of plasma on flux surfaces outside the edge. External kink modes may be unstable if they resonate with a rational surface in a vacuum region. They are stabilized by the presence of plasma at the rational surface. Resistivity at the rational surface may allow the instability to reappear with a much smaller growth rate as a tearing mode.

The presence of an axisymmetric poloidal divertor changes the situation in a fundamental way. Figure 2 shows the geometry in the presence of a divertor. (We specialize here to a single-null divertor.) The safety factor $q \rightarrow \infty$ as the divertor is approached. Plasma is present on every rational surface, although the rational surfaces are packed very closely together near the separatrix.

This purely axisymmetric picture is not realistic because the flux surfaces become increasingly fragile to break-up in the presence of nonaxisymmetry as we get closer to the separatrix. In reality, tokamaks are not perfectly axisymmetric. The effect of the magnetic nonaxisymmetry is to destroy the separatrix and nearby flux surfaces, allowing the field lines in that region to reach the divertor plate. We again recover a situation in which we have a nominal plasma edge having finite $q$, corresponding to the last good flux surface. The question arises whether the presence of plasma in the open field line region outside the last good flux surface can significantly affect the MHD stability of the tokamak.

It might naively be expected that the strong stabilizing influence obtained from the presence of plasma on the external rational surfaces in the non- 
diverted case would have no counterpart here. It might appear that there is nothing to distinguish one open field line from another, so that there can be no distinguished surface carrying a singular current. One point of this paper is to show that the complicated trajectories of the field lines in the open field line region defy this superficial intuition, defining singular surfaces that can carry large currents and constrain the motion of the plasma.

It has been recognized for some time that present day divertors display significant nonaxisymmetry. ${ }^{5}$ Several authors have studied the effects of nonaxisymmetric perturbations on the structure of a tokamak poloidal divertor. La Haye ${ }^{6}$ computed several field-line trajectories in the divertor region of the DIII-D tokamak; finding large changes in the trajectories in the presence of the field errors believed to exist on the device. Chu et al ${ }^{7}$ studied the feasibility of an X-point ergodic divertor. Pomphrey and Reiman ${ }^{1}$ employed a model magnetic field to determine the properties of the field line trajectories just outside the last good flux surface in a diverted tokamak. Although these field lines reach the divertor plate, it was found that they may execute many toroidal transits before doing so, so that there is a region of chaotic field line trajectories outside the last good flux surface. The footprint of the trajectories on the divertor plate was found to exhibit a coherent, spiral structure. Braams and Teytel ${ }^{8}$ have incorporated the effects of cross-field thermal conductivity in this picture to study the heat deposition on the divertor plate. Punjabi et $\mathrm{al}^{9,10}$ have modeled the divertor field with an area preserving map, and have used this model to study some aspects of nonaxisymmetric divertors.

The work described in this paper is related to that of Lau and Finn ${ }^{11}$ on three-dimensional reconnection of plasmoids in the solar corona and magnetosphere. The work of Lau and Finn, in turn, builds on the earlier work of Stern and of Greene on three-dimensional reconnection. ${ }^{2,3}$ Greene recognized that reconnection in three-dimensions is associated with the stable and unstable manifolds of unstable field nulls or unstable closed field lines. $\mathrm{He}$ focused his study on field nulls, which he argued are likely to be more important for space plasmas. Lau and Finn studied a periodic plasmoid as a model for a long solar coronal arcade. In this context, the reconnection is governed by an unstable closed field line. This is the relevant case for us in our study of the three-dimensional poloidal divertor.

The plan of the paper is as follows. In Sec. II we describe our model. 
For the axisymmetric piece of our field, we employ a simple divertor model consisting of two wires and a uniform toroidal field. The nonaxisymmetric piece of the field is specified analytically. In Sec. III we take advantage of the simplicity of the model, which allows rapid numerical evaluation of many field line trajectories over large distances. Plots of field line length as a function of initial coordinates exhibit singularities associated with the singular surfaces. An observed correspondence between the singularities and jumps in the number of poloidal transits of the orbits allows us to examine the structure of the singular surfaces using a contour plot of the number of poloidal transits as a function of the initial coordinates. The intersection of the singular surfaces with the divertor plate exhibits a spiral structure, establishing a connection with the work in Ref. 1 . We also obtain Poincare plots for near-resonant trajectories which have a large number of toroidal transits. Finally, in Sec. IV we discuss the implications of these results.

\section{The Divertor Model}

For the calculations described in this paper, we superpose an analytically specified nonaxisymmetric magnetic field on a simple two-wire divertor model. We work in cylindrical geometry, with the field a periodic function of $z$. Figure 3 shows our two-wire model for an axisymmetric divertor. A single, straight wire located at $x=0, y=1$ represents the plasma current. An additional straight wire located at $x=0, y=-1$ produces a single null divertor. For simplicity, the current in the two wires is taken to be equal. A divertor plate is located at $y=-0.5$.

The magnetic field for the unperturbed poloidal divertor can be written in the form

$$
\mathbf{B}=\hat{\mathbf{z}} \times \nabla \Psi_{0}+B_{0} \hat{\mathbf{z}},
$$

where

$$
\Psi_{0}=C \log \left(r_{p} r_{c}\right),
$$

$r_{p}$ and $r_{c}$ represent the distances from the two coils (as shown in the figure),

$$
\begin{aligned}
& r_{p}^{2}=x^{2}+(y-1)^{2}, \\
& r_{c}^{2}=x^{2}+(y+1)^{2}
\end{aligned}
$$

and $C$ is a constant that is proportional to the current in the coils. 
We superpose a nonaxisymmetric magnetic field,

$$
\delta \mathbf{B}=\epsilon B_{0} \nabla \chi,
$$

on our two-wire divertor model, with

$$
\chi=(1 / k) \exp (k x) \cos (k z) .
$$

This perturbation is manifestly curl-free. It is easily verified that it is also divergence-free.

To model tokamak geometry, we must introduce a periodicity length in the $z$ direction, $L=2 \pi R$, where we identify $R$ with the "major radius". Because we have normalized the distance from the wires to the X-point to 1 , $R$ is also roughly equal to the aspect ratio. We take $\mathrm{R}=3$ for our calculations. The nonaxisymmetric perturbation must satisfy

$$
k=n / R,
$$

where $n$ is an integer. We choose $C$ in Eq. (2) so that in the absence of the divertor coil the cylindrical safety factor, $q$, is equal to 3 on the $r_{p}=1$ surface. This gives

$$
C=B_{0} / 9 \text {. }
$$

The addition of the divertor coil produces an X-point at the origin, so that $q \rightarrow \infty$ there.

One conventional method of comparing field error magnitudes in tokamaks is to Fourier decompose the field error on a reference circular surface centered at the major radius. The Fourier components of Eq. (6) can be calculated from the expression ${ }^{12}$

$$
\exp (k \cos \theta)=\mathrm{I}_{0}(k)+\sum_{j=1}^{\infty} 2 \mathrm{I}_{j}(k) \cos (j \theta),
$$

where $I_{j}$ denotes the modified Bessel function of order $j$. For $n=1$, the $m=1, m=2$, and $m=3$ Fourier amplitudes of $\delta B / B$ are, respectively, $.49 \epsilon, .082 \epsilon$, and $.0069 \epsilon$. In present day tokamaks, the $n=1, m=2$ Fourier component of the field error is typically of order $10^{-4}$ times the toroidal field,$^{13-15}$ corresponding roughly to a value of $\epsilon=1.2 \times 10^{-3}$ in our model. 
To study the structure of the magnetic field, we integrate the field line equations,

$$
\begin{aligned}
& d x / d z=B^{x} / B^{z}, \\
& d y / d z=B^{y} / B^{z}
\end{aligned}
$$

with respect to $z$.

\section{Structure of the Open Field Lines}

In this section we explore the structure of the open field lines for the model described in Sec. II by employing numerical integration of the field line equations. The field line trajectories are initialized on the divertor plate, and they are followed until they again intersect the plate. We begin with an examination of the lengths of the field line trajectories as a function of their initial coordinates. This will lead us to the study of a set of trajectories having infinite length, which define a set of singular surfaces. We study the structure of the singular surfaces, and we uncover some interesting connections with previous work on three-dimensional divertors. ${ }^{1}$

Figure 4 is a plot of the value of the $z$-coordinate where a trajectory returns to the plate, $z_{f}$, as a function of the initial $x$-coordinate, $x_{0}$, in the absence of a perturbing field $(\epsilon=0)$. In constructing this figure we have followed 10,000 field line trajectories. The initial $z$ coordinate here is $z_{0}=0.0$. (For $\epsilon=0, z_{f}-z_{0}$ is of course independent of $z_{0}$.) For this plot we have focused on a narrow region near the separatrix which will show interesting structure for $\epsilon=10^{-3}$. For $\epsilon=0$ there is no additional fine-scale structure at any scale.

The singularity in Fig. 4 is of course associated with the divertor separatrix. The geometry is that shown in Fig. 3. The separatrix intersects the divertor plate at

$$
x=\left(2^{1 / 2}-5 / 4\right)^{1 / 2} \approx 0.4052 .
$$

A field line trajectory initiated at this value of $x$ remains on the portion of the separatrix between the plate and the X-line, asymptoting to the Xline as $z \rightarrow \infty$. (For $\epsilon=0$ the $\mathrm{X}$-line is independent of $z$, and we can refer interchangeably to the "X-point" or the "X-line".) As the initial x-coordinate of a trajectory approaches the separatrix, $z_{f} \rightarrow \infty$ logarithmically. 
In discussing the properties of the field line trajectories for $\epsilon>0$ we will refer to the "stable manifold" of the X-line. The stable manifold of an X-line is the set of points that are mapped asymptotically to the X-line by the field line trajectories. For $\epsilon=0$, the stable manifold coincides with a portion of the separatrix. (We regard the field line trajectories as being terminated by the divertor plate, so a portion of the separatrix maps onto the plate.)

It will be useful in the following to keep track of the number of times a trajectory goes around the tokamak in the poloidal direction. For this purpose, we define a quantity $n_{\theta}$ to be equal to the number of times that a trajectory crosses the $y=1$ plane from above. Figure 5 shows $n_{\theta}$ vs. $x_{0}$ (the initial $x$ coordinate) for $\epsilon=0$. Trajectories initialized just to the left of the point given by Eq. (12) lie in the private flux region and do not go around the plasma, so that $n_{\theta}=0$ for these trajectories. (See Fig. 2.) Trajectories which start to the right of this point go over the top of the plasma before returning to the divertor plate, so that $n_{\theta}=1$. There is a discontinuity in $n_{\theta}$ at the point where the separatrix intersects the divertor plate, corresponding to a change in field line topology. Field lines on either side of the separatrix are continuously deformable to nearby field lines. (This involves a small motion of the foot-point.) On the other hand, a field line just to one side of the separatrix cannot be deformed in a continuous manner into one on the other side of the separatrix.

Next we introduce a three-dimensional magnetic field perturbation corresponding to $\epsilon=0.001$. This value of $\epsilon$ is chosen to roughly correspond to the magnitude of field error in many present day tokamaks. It is also roughly equal to the nonaxisymmetric magnetic field perturbation amplitude used in many of the calculations of Ref. 1 . Figure 6 is a plot of $z_{f}$ vs. $x_{0}$ for this value of $\epsilon$. We have again followed 10,000 field line trajectories. We see that the singularity at the separatrix has broken up into a complicated structure displaying multiple singularities. This can be understood if we recall that the flux surfaces just inside the separatrix break up in the presence of a threedimensional perturbation. Associated with each broken rational surface is a set of closed orbits corresponding to unstable fixed points (X-lines). Field line trajectories in the region of broken surfaces intersect the divertor plate, as do stable manifolds of the X-lines in this region. (Although the field lines that lie in remnant islands do not intersect the divertor plate.) At each such intersection of a stable manifold with the divertor plate, $z_{f}$ must go to $\infty$. 
Figure 7 is a plot of $n_{\theta}$ vs. $x_{0}$ for $\epsilon=0.001$. For $x_{0}<.403, n_{\theta}=0$, as before, corresponding to the region of private flux. For $x_{0}>.407, n_{\theta}$ is also unchanged from the $\epsilon=0$ case, corresponding to field lines which go over the top of the plasma and then return to the divertor plate. Intermediate values of $x_{0}$ have higher values of $n_{\theta}$. The orbits are temporarily "captured", and circle the plasma multiple times before returning to the divertor plate. We can begin to make contact here with previous work on nonaxisymmetric divertors. ${ }^{1}$ In the presence of a nonaxisymmetric perturbation, field line trajectories in the region of broken flux surfaces define a region of chaotic field lines that can circle the plasma many times before escaping to the plate. The $n_{\theta}>0$ trajectories in Fig. 7 are temporarily captured by this chaotic region.

There are discontinuities in the $n_{\theta}$ vs. $x_{0}$ plot, corresponding to the borders between regions where the field lines circle the plasma different numbers of times. These borders define three-dimensional singular surfaces separating regions of differing topology. At the intersection of such a singular surface with the divertor plate, $z_{f} \rightarrow \infty$, as can be observed in Fig. 6 .

We have thus far been following field lines that intersect the divertor plate at $z=0$. To study the toroidal structure of the singular surfaces, we make use of the fact that they correspond to jumps in $n_{\theta}$. In Fig. 8 we have plotted the contours separating different values of $n_{\theta}$ in polar coordinates. For reference, the location of the intersection of the unperturbed separatrix with the plate (at $x_{0} \approx .405$ ) is shown as a dashed circle. The angular coordinate for the plot is $\phi=z / R$. Since the width of the structure is small compared to its radial location, we have used $x_{0}-x_{r}$, with $x_{r} \approx .4025$, as the radial variable for the polar plot. Subtracting off a reference value of $x$ in this way allows us to choose a radial scale that magnifies the structure. The contours separating values of $n_{\theta}$ from 0 to 12 have been plotted. The intersection of the singular surfaces with the divertor plate takes the form of a spiral structure. (The roughness that can be observed in the contours appears to be caused by the fact that the plotter is interpolating between integer values of $n_{\theta}$ evaluated on a discrete grid, and should not be taken to be indicative of any lack of differentiability. The roughness is observed to scale with the fineness of the grid. For the plot shown, a $200 \times 200$ grid in $x_{0}-z_{0}$ coordinates was used, and 40,000 field line trajectories were computed.) Scrape-off layer widths are typically of the order of $1 \%$ of the minor radius, and the width of the spiral structure is roughly comparable to this for the perturbation amplitude 
chosen.

The spiral structure of Fig. 8 is similar to that observed in a different context in Ref. 1. In Ref. 1, the footprint of a set of set of field lines on the divertor plate was calculated as a first approximation to the heat deposition pattern on the plate in the presence of a nonaxisymmetric field. A set of 2000 field line trajectories was initialized just outside the last good flux surface, and the trajectories were followed until they intersected the divertor plate. The footprint defined by the intersections with the plate was found to have a spiral structure. Smaller scale local structure was also visible in the footprint, perhaps reflecting the influence of the singular surfaces.

The identification of the singular surfaces with the stable manifolds of $\mathrm{X}$-lines suggests the presence of a fractal structure. The break-up of the flux surfaces near the separatrix leads to the formation of an infinite hierarchy of $\mathrm{X}$-lines, each having an associated stable manifold.

To investigate the small scale structure of our singularities, we magnify the region near the leftmost peak of Fig. 6. Figure 9 is a plot of $z_{f}$ vs. $x_{0}$ for $x_{0}$ in the range from .40365 to .40375 . What appears as a single, narrow peak in Fig. 6 reveals a complex structure when magnified in this way. Notice the difference in the $z_{f}$ scales for the two plots.

In Figure 10 we magnify the $x_{0}$ scale even further, again focusing on the leftmost peak. Here $x_{0}$ ranges from .403662 to .4036635 , corresponding to the leftmost peak in Fig. 9. Figures 9 and 10 show evidence of a self-similar structure.

The behavior we have been describing in this section corresponds to chaotic scattering of the field line trajectories. (See e.g. Ref. 16.) Similar behavior can arise in the scattering of a particle off a classical potential. Depending on its energy and impact parameter, a particle which comes in from infinity may be captured by the potential and orbit indefinitely. Analogous to our plots of $z_{f}$, one may define a "delay time" for this case to be the time it takes the particle to exit some region. For those trajectories that are captured by the potential, the delay time goes to infinity. A plot of delay time vs. impact parameter shows a singular structure similar to that in our plot of $z_{f}$ vs. $x_{0}$. A plot of the net deflection in a particles orbit as a function of impact parameter shows singularities coinciding with the singularities in the plot of the delay time. The singularities have a fractal structure. Magnification of the neighborhood of one of the peaks reveals further structure. 
As the neighborhoods of newly revealed peaks are in turn magnified, a selfsimilar structure becomes apparent, with the structure repeating itself on smaller and smaller scales.

The field line structure we have described is similar to that observed by Lau and Finn in the context of three-dimensional reconnection of plasmoids in the solar corona and magnetosphere. ${ }^{11}$ Studying periodic perturbations of solar arcades having a separatrix, they observed a fractal singularity structure in plots of the field line lengths as a function of footpoint location, and they made the connection with chaotic scattering.

Finally, it is interesting to look at Poincare plots for some of our trajectories. A Poincare plot shows the intersection of a trajectory with $z=2 n \pi R$, $n$ an integer. By and large our field line trajectories return rapidly to the divertor plate, where they are terminated, and have at most a few intersections with these surfaces. The Poincare plot for such a trajectory consists of only a few points, and is not very interesting. We can, however, usefully look at Poincare plots for the trajectories having large $z_{f}$. Two such plots for $\epsilon=10^{-3}$ are shown in Fig. 11 and Fig. 12. The trajectory associated with Fig. 11 has $x_{0} \approx 0.4045$, with $z_{f} \approx 3114$ and $n_{\theta}=108$. Note the $m=3$ structure, suggesting that the nearby stable manifold is associated with an $m=3 \mathrm{X}$-line. The trajectory of Fig. 12 corresponds to $x_{0} \approx .40367$, near the left edge of the region shown in Fig. 6 . It has $z_{f} \approx 3233$ and $n_{\theta}=118$. The appearance of the Poincare plot suggests that the nearby stable manifold is associated with the $m=0$ divertor X-line.

\section{Discussion}

Conventional models of tokamak divertors assume axisymmetry. In practice, there is always some nonaxisymmetry present. The separatrix is very sensitive to magnetic field perturbations, so that even relatively small perturbations will destroy the separatrix and a significant region of nearby magnetic surfaces, producing a region of open field lines there. In present day tokamaks, nonaxisymmetric "error fields" are typically of order $\delta B / B \approx$ $10^{-3} \cdot{ }^{13-15}$ This perturbation is sufficiently large to open up the magnetic surfaces in a region whose width is comparable to that of the scrape-off layer. ${ }^{1}$ Similarly, perturbations arising from the nonlinear evolution of free-boundary MHD instabilities, or externally imposed for the express purpose of influenc- 
ing the edge behavior of the plasma, will have a significant effect on the magnetic field structure near the separatrix.

In an axisymmetric diverted tokamak, the divertor separatrix forms a barrier which prevents open field lines from executing multiple poloidal transits around the tokamak. In the terminology of Sec. III, the open field lines in this case must have either $n_{\theta}=0$ or $n_{\theta}=1$. (See figures 2 and 5.) A nonaxisymmetric perturbation changes this situation, breaking the separatrix and allowing open field line trajectories starting at the divertor plate to circle the tokamak in the poloidal direction multiple times before returning to the divertor plate $\left(n_{\theta}>1\right)$. Field lines which circle the plasma different numbers of times (have different values of $n_{\theta}$ ) are topologically distinct. The boundaries between regions of differing $n_{\theta}$ define singular surfaces. Figure 8 shows the intersection of a set of singular surfaces with the divertor plate for our model field.

A field line in the interior of a region having a given value of $n_{\theta}$ can be subject to small magnetic perturbations without changing its topology. On the other hand, for a field line very close to a singular surface, a small perturbation of the magnetic field can cause reconnection. Referring to Fig. 2, consider the open field lines in the "common flux" region outside the separatrix, which go over the top of the tokamak. If a field line is far from the separatrix, all of the neighboring field lines have the same topology, and the topology of the field line is invariant under small perturbations of the magnetic field. If the field line is close to the separatrix, on the other hand, an appropriate perturbation of the magnetic field can cause it to pinch off at the X-point and separate into two field lines, a closed line which moves into the interior of the tokamak, and an open field line which moves into the region of private flux.

In ideal MHD, the magnetic field lines can be viewed as moving with the velocity of the fluid, and their topology must be preserved. ${ }^{17}$ Magnetic field perturbations are constrained by the requirement that they preserve the field line topology, with surface currents appearing at singular surfaces to shield out perturbations that would cause reconnection there.

The presence of a small but finite resistivity causes surface currents to decay, allowing the constraints of ideal MHD to be broken on a longer time scale. Reconnection takes place. In a three-dimensional divertor, perturbations on the ideal MHD time scale give rise to localized currents at the 
singular surfaces. On a longer time scale, reconnection can take place at the singular surfaces.

This intuitive picture can be related to the more rigorous picture of threedimensional reconnection that has been developed in the context of space plasmas. ${ }^{2-4,18}$ The approach of Stern $^{2}$ and Greene ${ }^{3}$ has been to consider the $\mathbf{E} \times \mathbf{B}$ motion of the field lines produced by an electrostatic potential, $\mathbf{E}=$ $-\nabla \phi$. The electrostatic potential must be constant along field lines, $\mathbf{B} \cdot \nabla \phi=$ 0 . In the presence of closed field lines or field nulls, the solutions to this magnetic differential equation, subject to appropriate boundary conditions, develop discontinuities. Delta functions appear in the solution for the electric field and fluid velocity. This is taken to be a signature for the appearance of reconnection. It is argued that reconnection in three-dimensions is associated with either closed field lines or field nulls. The delta functions lie along the stable and unstable manifolds of the closed field lines and nulls, so that is where we can expect to see sheet currents due to reconnection.

There are no field nulls in our case, so the theory of Stern and Greene predicts that reconnection is associated with X-lines. Singular currents form on the stable and unstable manifolds of the X-lines. These are the surfaces where $z_{f} \rightarrow \infty$. We have seen that the singularities of $z_{f}$ coincide with the discontinuities of $n_{\theta}$, that is, with the discontinuities in field line topology.

The picture of Stern and Greene has been extended to include an externally imposed time dependent vector potential by Lau and Finn. ${ }^{4}$ Lau and Finn observe that, if flux surfaces exist, the $\mathbf{E} \times \mathbf{B}$ velocity driven by an electrostatic potential lies entirely in the flux surfaces. To recover the conventional picture of two-dimensional reconnection, where field lines move across the flux surfaces into a separatrix, they add an externally imposed inductive component to the electric field, $\mathbf{E}=-\nabla \phi-\partial \mathbf{A} / \partial t$. The condition that $\mathbf{E} \cdot \mathbf{B}=0$ gives them a magnetic differential equation for $\phi$ :

$$
\mathbf{B} \cdot \nabla \phi=-\mathbf{B} \cdot \frac{\partial \mathbf{A}}{\partial t} \text {. }
$$

Again, the presence of closed field lines or field nulls leads to the appearance of singularities in the solution for $\phi$. The case of interest for us is that where closed field lines are present. The singularities again lie along the stable and unstz.je manifolds of the X-lines. As we approach the stable and unstable manifolds the flow velocity becomes very large, signaling the local breakdown of the theory and the need to bring in some additional piece of physics, such 
as resistivity. This is the locus of reconnection. A more discursive exposition of this picture can be found in Ref. 18.

We have argued that unconstrained magnetic field perturbations at the singular open surfaces can lead to reconnection. This implies the existence of constraints on the self-consistent magnetic field perturbations that can appear at these surfaces on an ideal MHD time scale. The constraints have implications for free-boundary MHD instabilities, such as external kinks. To the extent that edge localized modes (ELMs) are external modes (e.g. localized external kinks), they too will be influenced by the singular surface constraints.

The singular surface constraints limit the free-energy that can be tapped by ideal MHD instabilities, and are therefore stabilizing. Instabilities which are stabilized on the ideal time scale may reappear as tearing modes on a longer time scale, as the resistivity relaxes the constraints and allows the plasma access to an additional reservoir of free energy. The tearing is localized at the singular surfaces.

For external modes rotating with the plasma, the stationary field lines in the scrape-off layer see an external perturbation with real frequency. In this case, localized currents continue to be induced at the singular surfaces on long time scales. Stabilization persists into the resistive time scale. This effect is well know in the context of stabilization by a resistive wall, where plasma rotation relative to the wall allows the stabilizing wall currents to persist. ${ }^{19,20}$

The open singular surfaces will play an important role in the nonlinear evolution of a tearing mode whose rational surface is close to the divertor separatrix. When the tearing mode reaches sufficient amplitude, its rational surface self-consistently opens up. In the absence of the constraint associated with the open singular surface, the mode would enter a phase of rapid growth.

The stabilizing constraints at the singular surfaces are enforced by localized currents which shield out perturbations whose presence would cause reconnection. It is the resistive decay of these localized currents that may remove the stabilizing constraint on longer time scales. The time scale on which they decay, and therefore the effectiveness with which they resist perturbations will be affected by how localized the stabilizing currents need to be, which is in turn affected by the field line structure. If the chaotic nature of the field lines near the singular surface cause the induced currents 
to rapidly dissipate after broadening only slightly, that will reduce the time scale on which the induced currents can provide effective stabilization. Similarly, if the induced stabilizing currents are largely confined to a narrow region around the $\mathrm{X}$-line, and are small elsewhere on the singular surface, that will also reduce their effectiveness. This issue of time scales is outside the scope of this paper.

It is natural to ask whether some external control could be exerted over the localized currents to extend their influence to longer time scales and to more effectively stabilize deleterious modes. For the analogous currents at toroidal rational surfaces, it has been proposed that current drive ${ }^{21}$ or localized heating ${ }^{22}$ could be used for that purpose. Similarly, feedback stabilization using external coils can be regarded as an artificial way of imposing stabilizing currents that would arise naturally if a conducting wall were present.

Because the open singular surfaces intersect the divertor plate, we can drive currents in the neighborhood of these surfaces directly. This may be accomplished either by application of an electrical bias potential, or through the use of thermionic emission. ${ }^{23}$ The lengths of the field line trajectories get large near a singular surface, so the resistance along the field lines gets large. A large, radially localized voltage is needed.

The direct access to the open singular surfaces also suggests the possibility for obtaining useful diagnostic information. When the singular surface constraints stabilize an otherwise unstable mode, localized currents are induced at the singular surfaces. The induced currents impinge directly on the divertor plates, where they can be measured. Very localized measurements would be necessary to extract the full structure of the induced current for a nonrotating mode. If the mode rotates, adequate resolution in the time domain could be substituted for resolution in the toroidal direction. Even measurements of relatively low resolution should be capable of distinguishing the spiral current distribution implied by Fig. 8 from a sinusoidal current distribution. Measurement of the induced curents on the singular surfaces is of potential interest as a method for early detection of imminent disruptions.

A comment is necessary concerning the issue of the net current flowing into the divertor plate. This becomes an issue if we recall that, unlike our stylized model of Fig. 3, the inner and outer strike-points of the unperturbed separatrix may in practice lie on different divertor plates. In that case, the 
two ends of each of the open field lines lie on different plates. If the net current flowing into a plate is nonzero, the plate will rapidly charge up, and the resulting electric field will drive a diffuse return current that cancels the net current. A diffuse return current will not affect any of the conclusions of this section. In general the imposed perturbation is sinusoidal, so the net induced current will be relatively small, and the resulting diffuse return current will also be small.

Finally, we note that in the presence of the rapid motion associated with a disruption, large nonaxisymmetric halo currents will be induced along the singular open surfaces, with corresponding torques on the external coils and localized damage on the divertor plates.

\section{ACKNOWLEDGMENT}

I am grateful for useful discussions with A. Boozer, J. Finn, J. Greene and N. Pomphrey. Some of the computer programming for the work described in this paper was done by Kumaran Damodaran as an NUF summer student at the Princeton Plasma Physics Laboratory. This work was supported by DoE contract No. DE-AC02-76-CHO-3073. 


\section{References}

${ }^{1}$ N. Pomphrey and A. Reiman, Phys. Fluids B 4(4), 938-948 (April 1992).

${ }^{2}$ D. P. Stern, J. Geophys. Res. 78, 7292-7305 (1973).

${ }^{3}$ J. M. Greene, J. Geophys. Res. 93(A8), 8583-8590 (August 1988).

${ }^{4}$ Y. Lau and J. Finn, Ap. J. 350, 672-691 (February 1990).

${ }^{5}$ T. E. Evans, J. Neuhauser, F. Leuterer, E. R. Mueller, and the Asdex Team, in Proceedings of the Ninth International Conference on Plasma Surface Interactions, May 20-25, 1990.

${ }^{6}$ R. LaHaye, Nuclear Fusion 31, 1550-1555 (August 1991).

${ }^{7}$ M. S. Chu, T. H. Jensen, R. J. L. Haye, T. S. Taylor, and T. E. Evans, Technical Report GA-A20682, General Atomics, San Diego, Calif., October 1991.

${ }^{8}$ B. J. Braams and M. D. Teytel, 1993, Private communication and paper 1C21, 1993 International Sherwood Fusion Theory Conference.

${ }^{9}$ A. Punjabi, A. Verma, and A. Boozer, Physical Review Letters 69, 33223325 (December 1992).

${ }^{10}$ A. Punjabi, A. Verma, and A. Boozer, J. Plasma Physics 52, 91-111 (1994).

${ }^{11}$ Y. Lau and J. Finn, Ap. J. 366, 577-591 (January 1991).

${ }^{12}$ M. Abramowitz and I. Stegun, editors, Handbook of Mathematical Functions, chapter 9, page 376, National Bureau of Standards, Washington, D. C., 1964.

${ }^{13}$ J. A. Snipes, D. J. Campbell, T. C. Hender, M. V. Hellermann, and H. Weisen, Nucl. Fusion 30(2), 205-218 (1990).

${ }^{14}$ H. Zohm, A. Kallenbach, H. Bruhns, G. Fussmann, and O. Klueber, Europhys. Lett. 11(8), 745-750 (1990). 
${ }^{15}$ R. J. LaHaye and J. T. Scoville, Review of Scientific Instruments 62, 2146-2153 (September 1991).

${ }^{16} \mathrm{~S}$. Bleher, C. Grebogi, and E. Ott, Physica D 46, 87-121 (1990).

${ }^{17}$ W. Newcomb, Annals of Physics 3, 347-385 (1958).

${ }^{18}$ D. Biskamp, Nonlinear Magnetohydrodynamics, Cambridge University Press, 1993.

${ }^{19}$ M. F. F. Nave and J. A. Wesson, in Controlled Fusion and Plasma Physics (Proc. 14th Eur. Conf. Madrid, 1987), Vol. 11D, Pt. III, page 1103, European Physical Society, 1987.

${ }^{20}$ T. C. Hender, C. G. Gimblett, and D. C. Robinson, in 15th European Conference on Controlled Fusion and Plasma Heating, edited by S. Pesic and J. Jacquinot, pages 437-440, Dubrovnik, May 1988, European Physical Society, Volume 12B, part I.

${ }^{21}$ A. H. Reiman, Phys. Fluids 26, 1338-1340 (May 1983).

${ }^{22}$ Y. Yoshioka, S. Kinoshita, and T. Kobayashi, Nucl. Fusion 24, 565 (1984).

${ }^{23}$ J. Kesner, J. J. Ramos, and S. C. Luckhardt, Nucl. Fusion 34(6), 795-801 (1994). 


\section{Figures}

FIG. 1. Geometry of a non-diverted tokamak (poloidal cross-section). Dashed lines indicate interior flux surfaces, and solid lines indicate exterior flux surfaces.

FIG. 2. Geometry of tokamak magnetic field lines in the presence of an axisymmetric poloidal divertor.

FIG. 3. Two-wire model for an axisymmetric divertor. $I_{p}$ and $I_{c}$ indicate the current filaments used in the model. At any given point, $r_{p}$ and $r_{c}$ represent the distances from the filaments. The field lines shown are those produced by taking equal currents in the two filaments.

FIG. 4. Distance that field lines travel in the $z$ direction before again hitting the divertor plate, $z_{f}$, plotted as a function of the initial $x$ coordinate, $x_{0}$. Initial points of the trajectories lie on the plate. Perturbing field is absent $(\epsilon=0)$.

FIG. 5. Number of times that the trajectory crosses the $x=1$ plane from above, $n_{\theta}$, plotted as a function of the initial $x$ coordinate, $x_{0} . \epsilon=0$.

FIG. 6. Distance that field lines travel in the $z$ direction for $\epsilon=0.014$.

FIG. 7. $n_{\theta}$ vs. $x_{0}$ for $\epsilon=0.014$

FIG. 8. Contours separating different values of $n_{\theta}$ as a function of $x_{0}$ and $z_{0}$.

FIG. 9. Blow-up of the leftmost peak in Fig. 6.

FIG. 10. Blow-up of the leftmost peak in Fig. 9.

FIG. 11. Poincare plot for the trajectory initialized at $x_{0}=0.404503714$.

FIG. 12. Poincare plot for the trajectory initialized at $x_{0}=0.4036724736842$. 


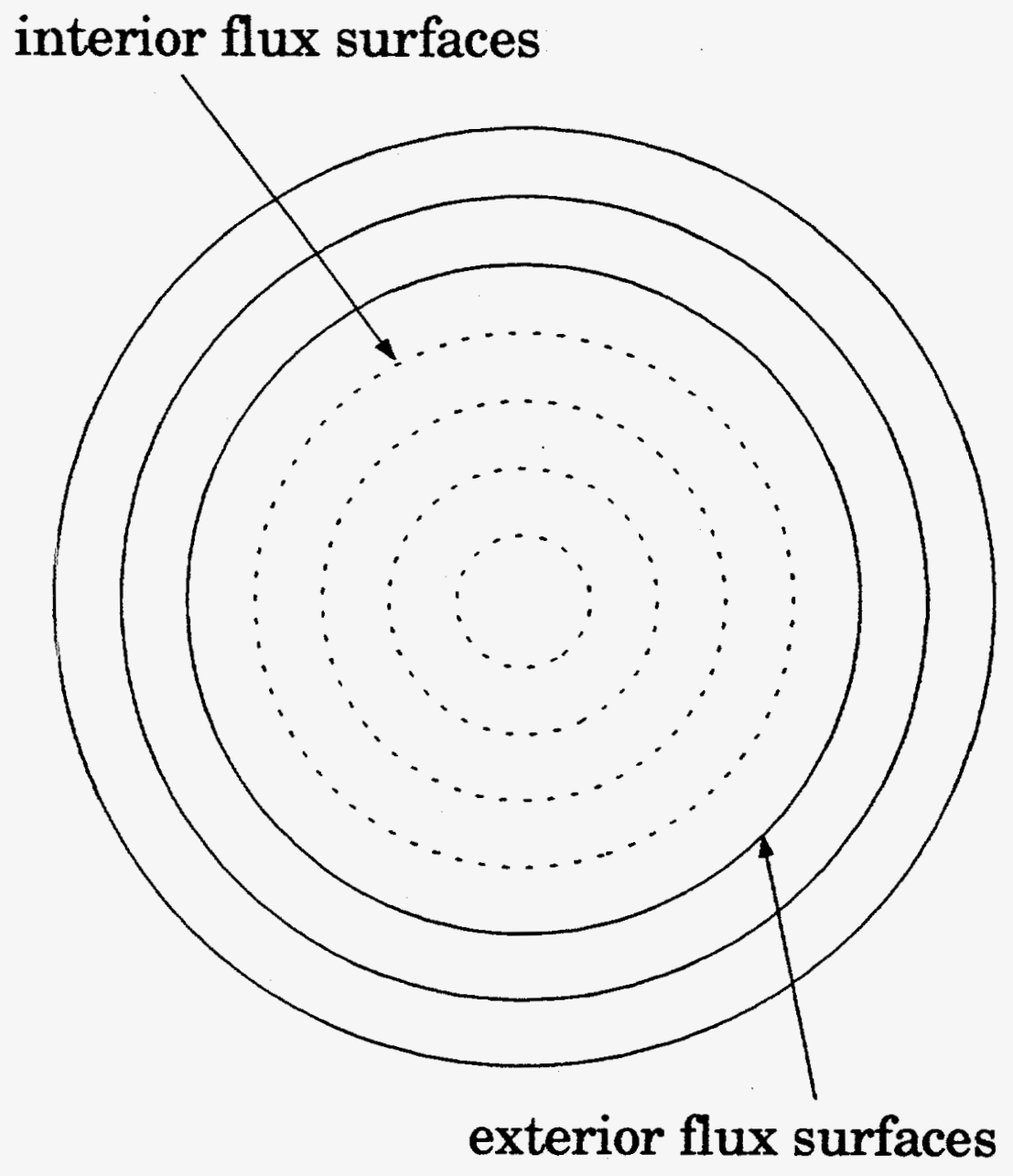

Fig. I 


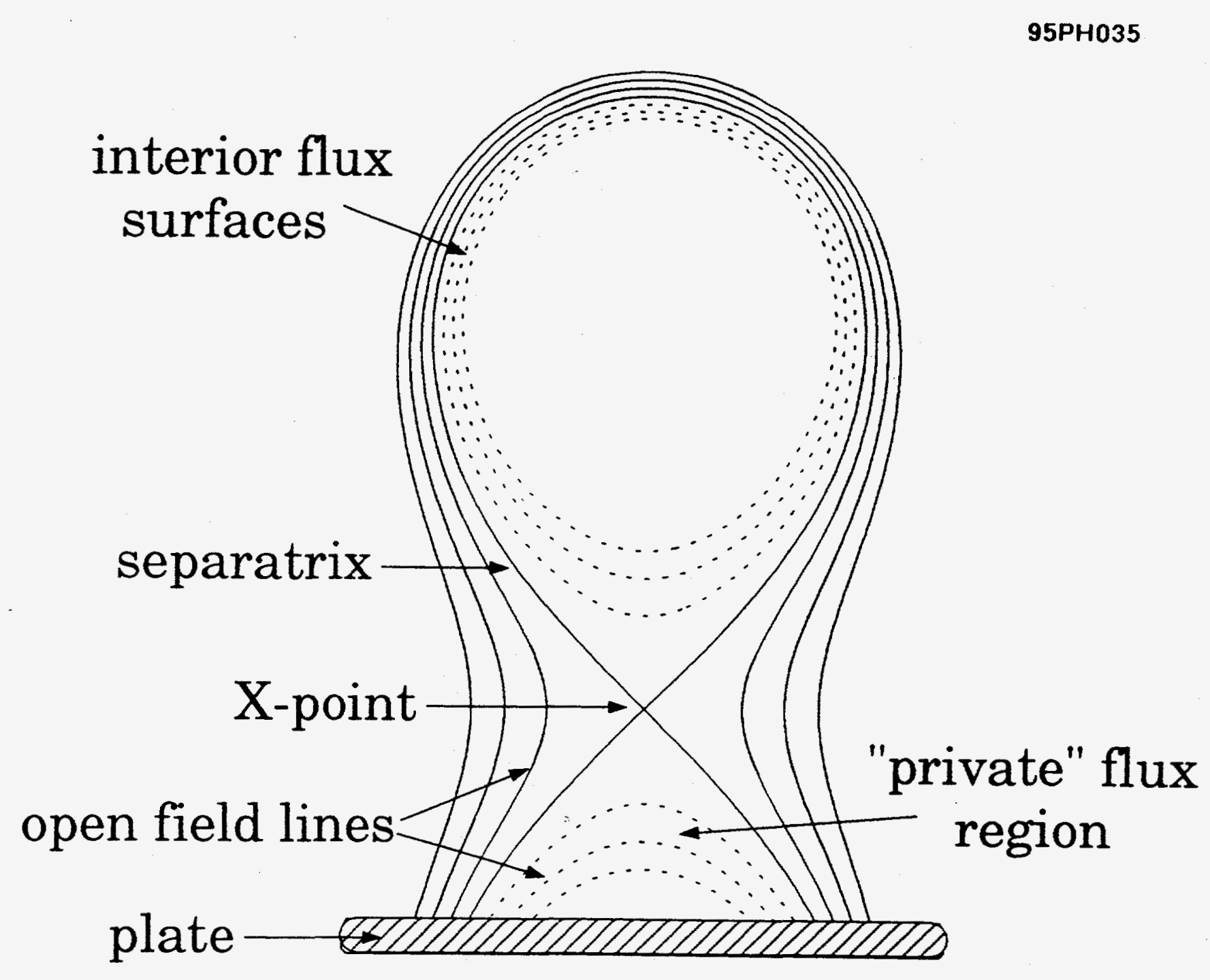

Fig. 2 
95PH037

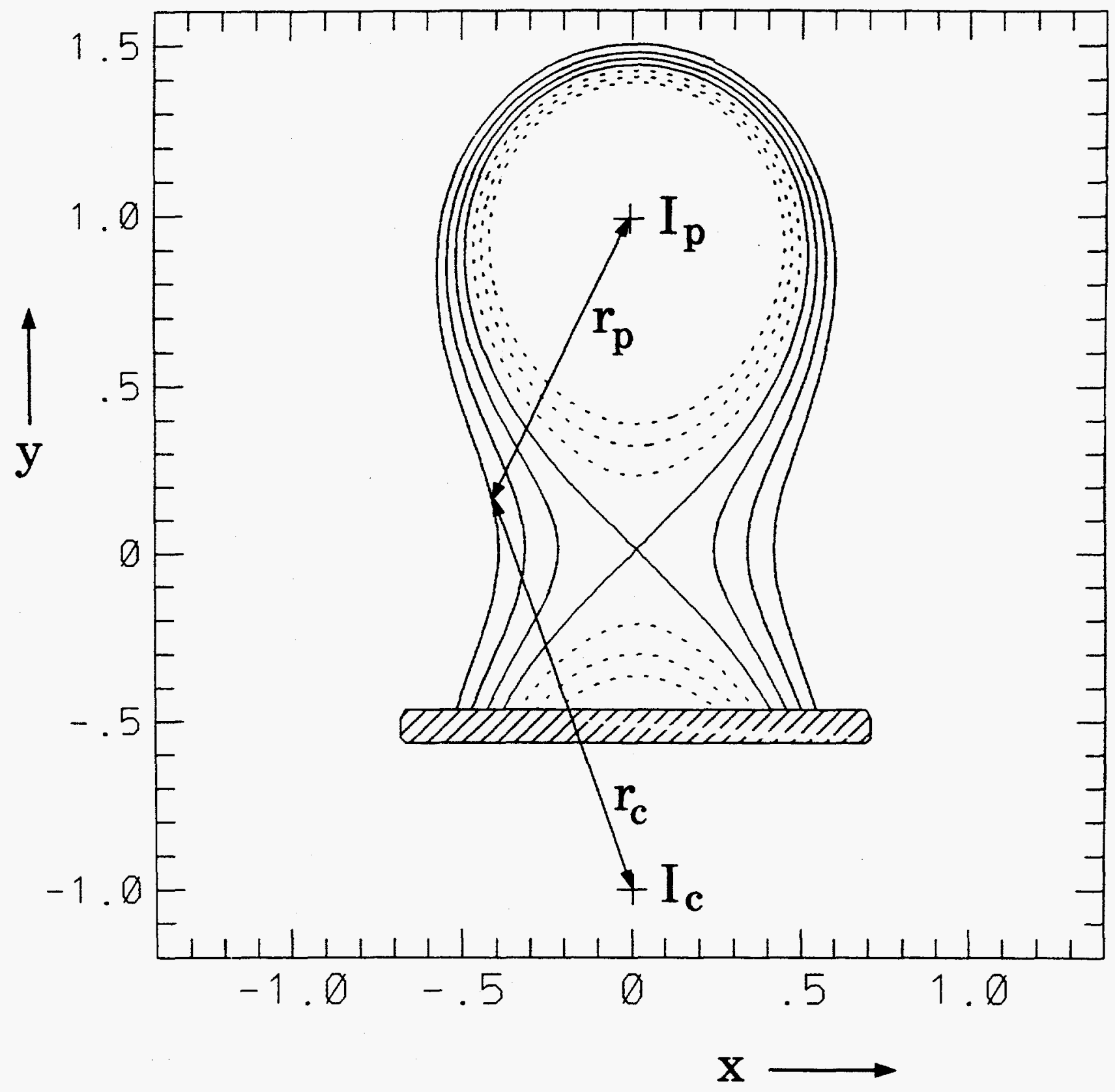

Fig. 3 
95PH038

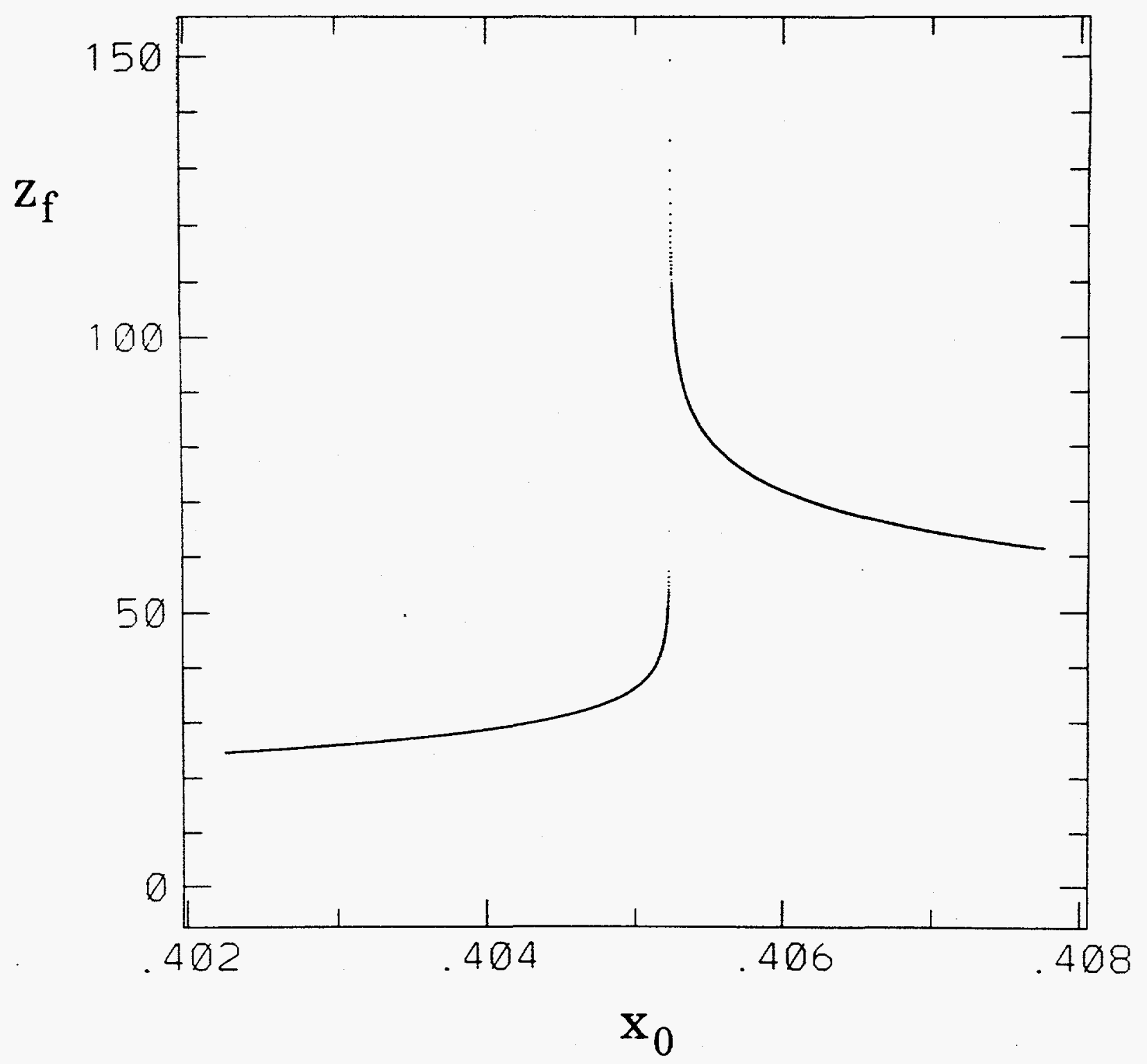

Fig. 4 
95PH039

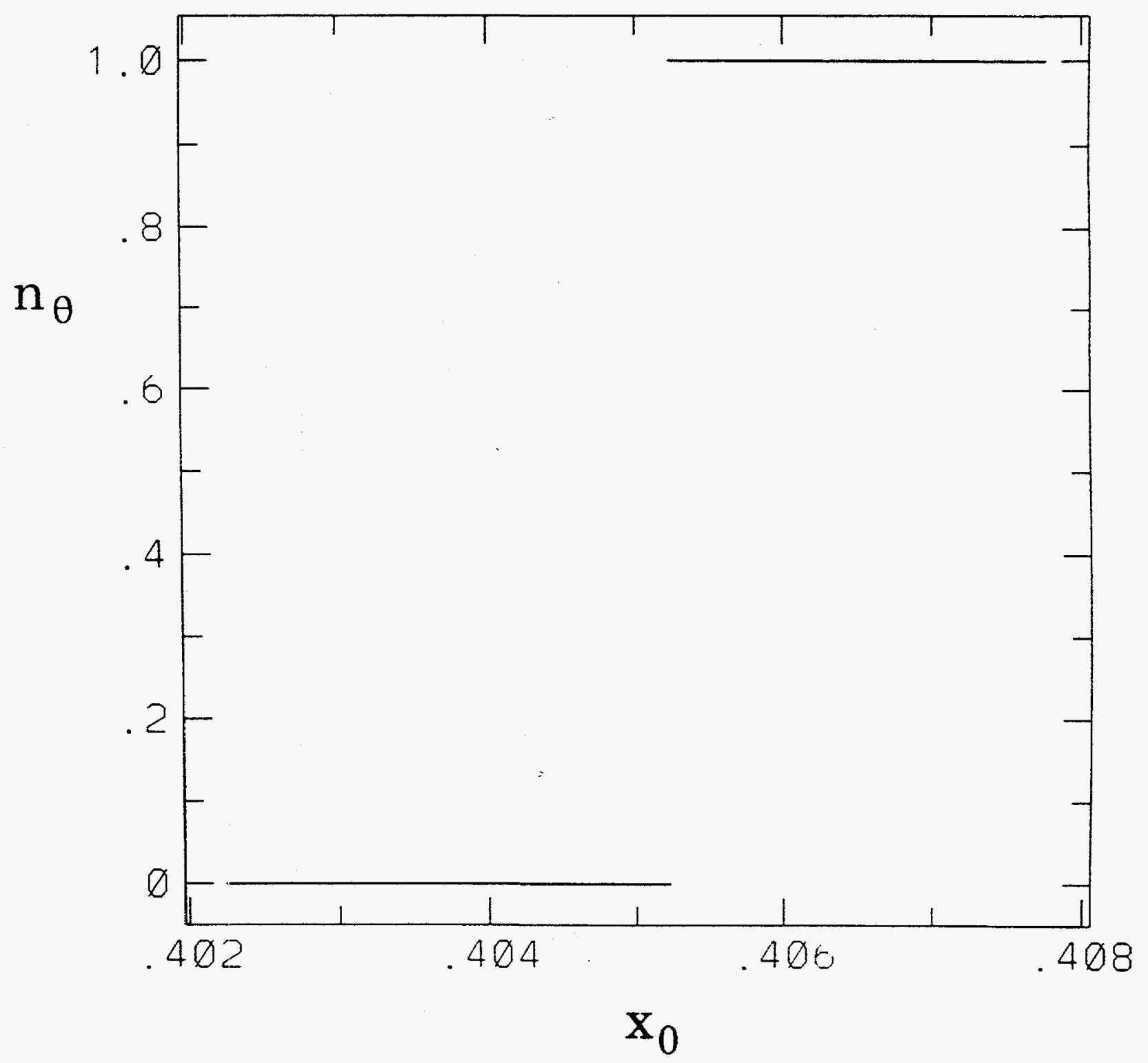

Fig. 5 
95PH040

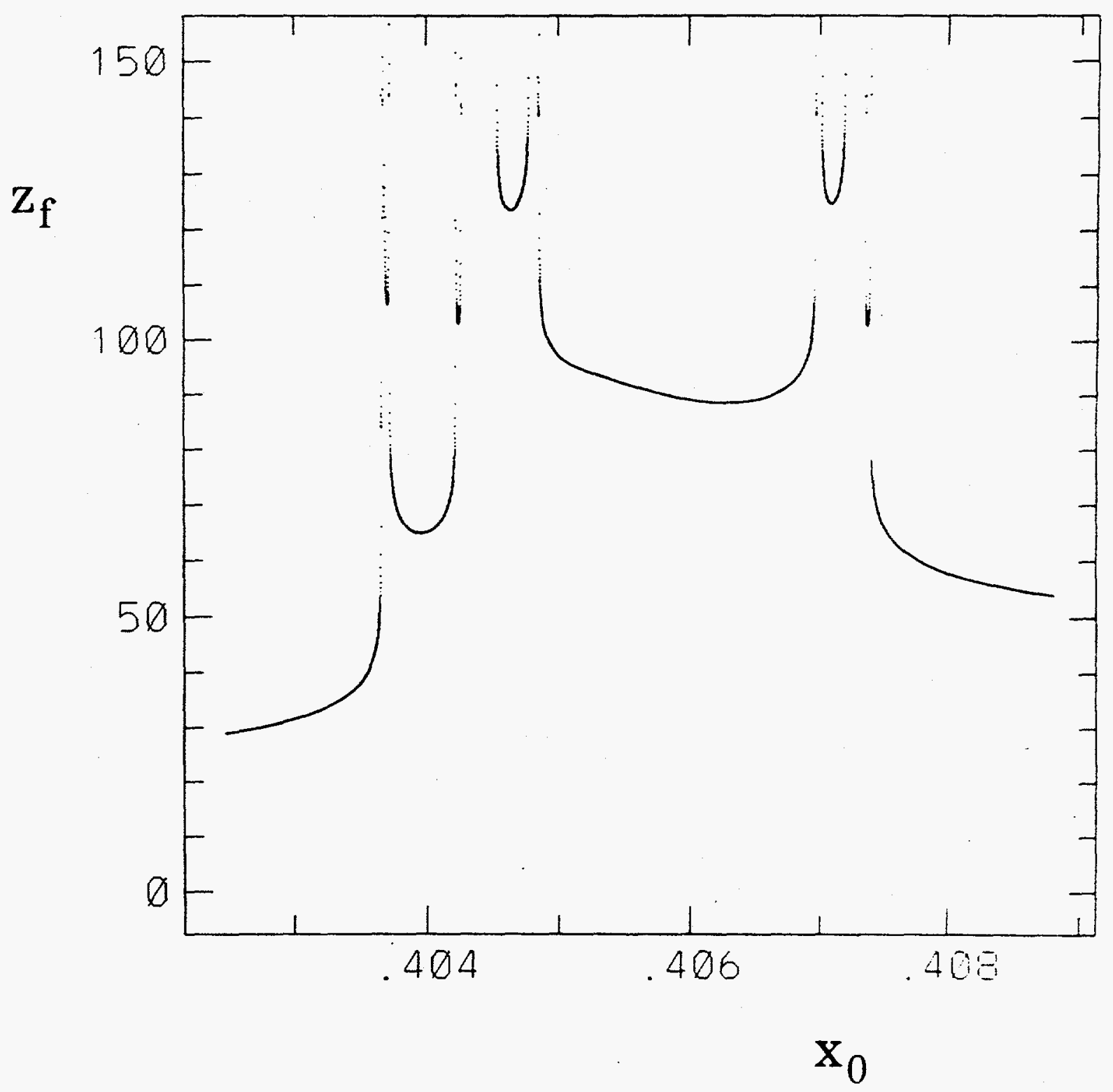

Fig. 6 
95PH041

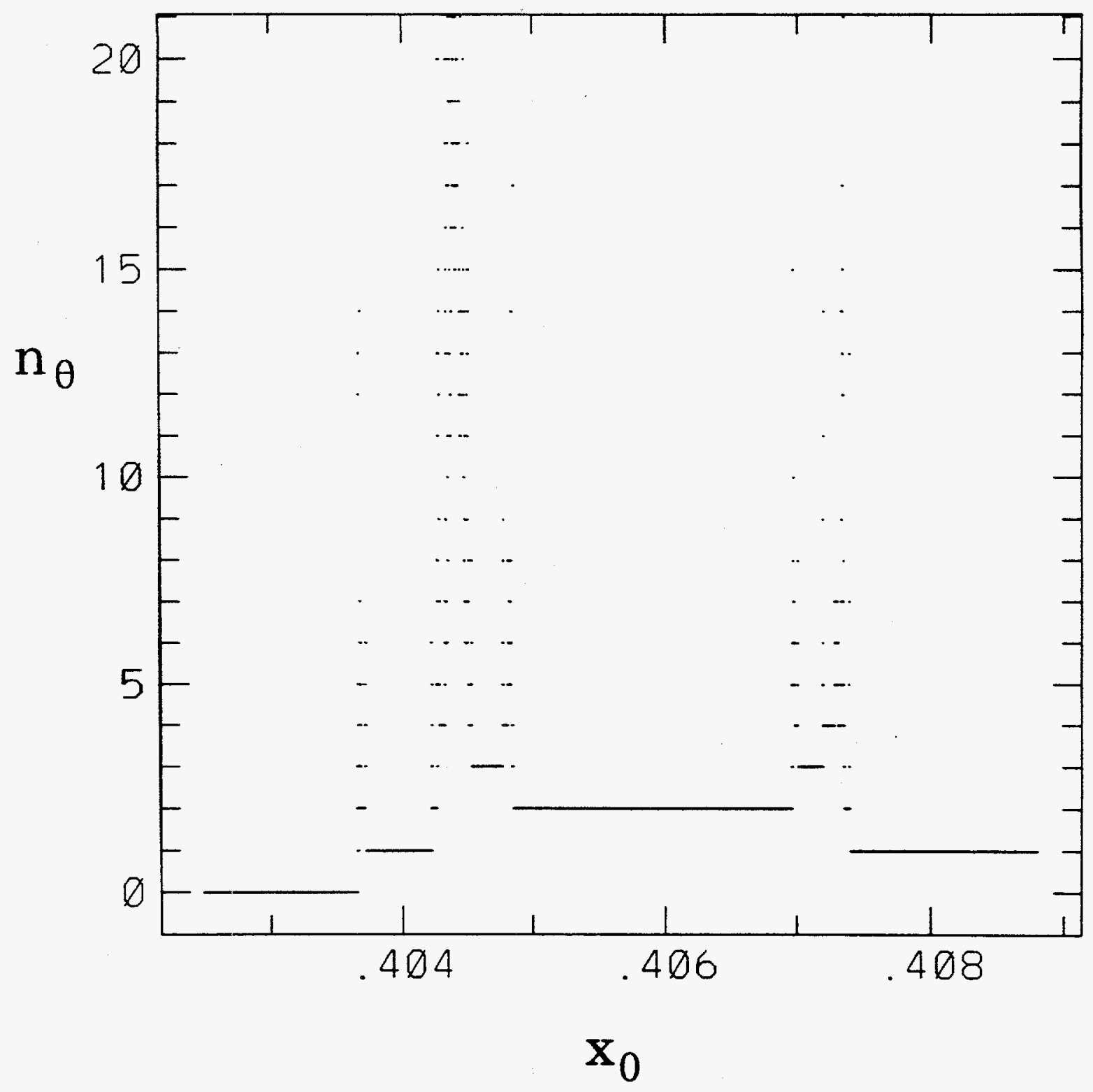

Fig. 7 


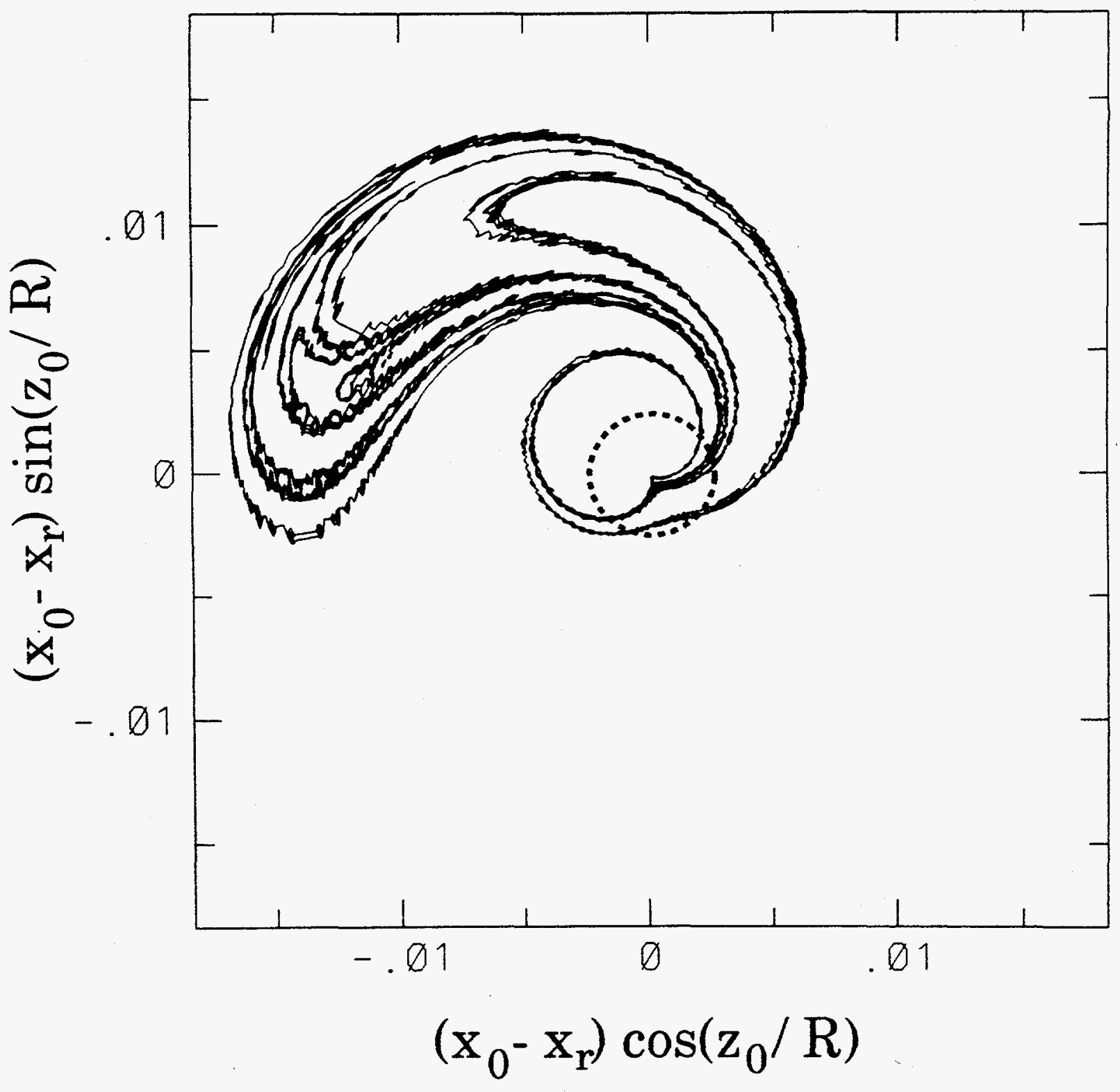

Fig. 8 


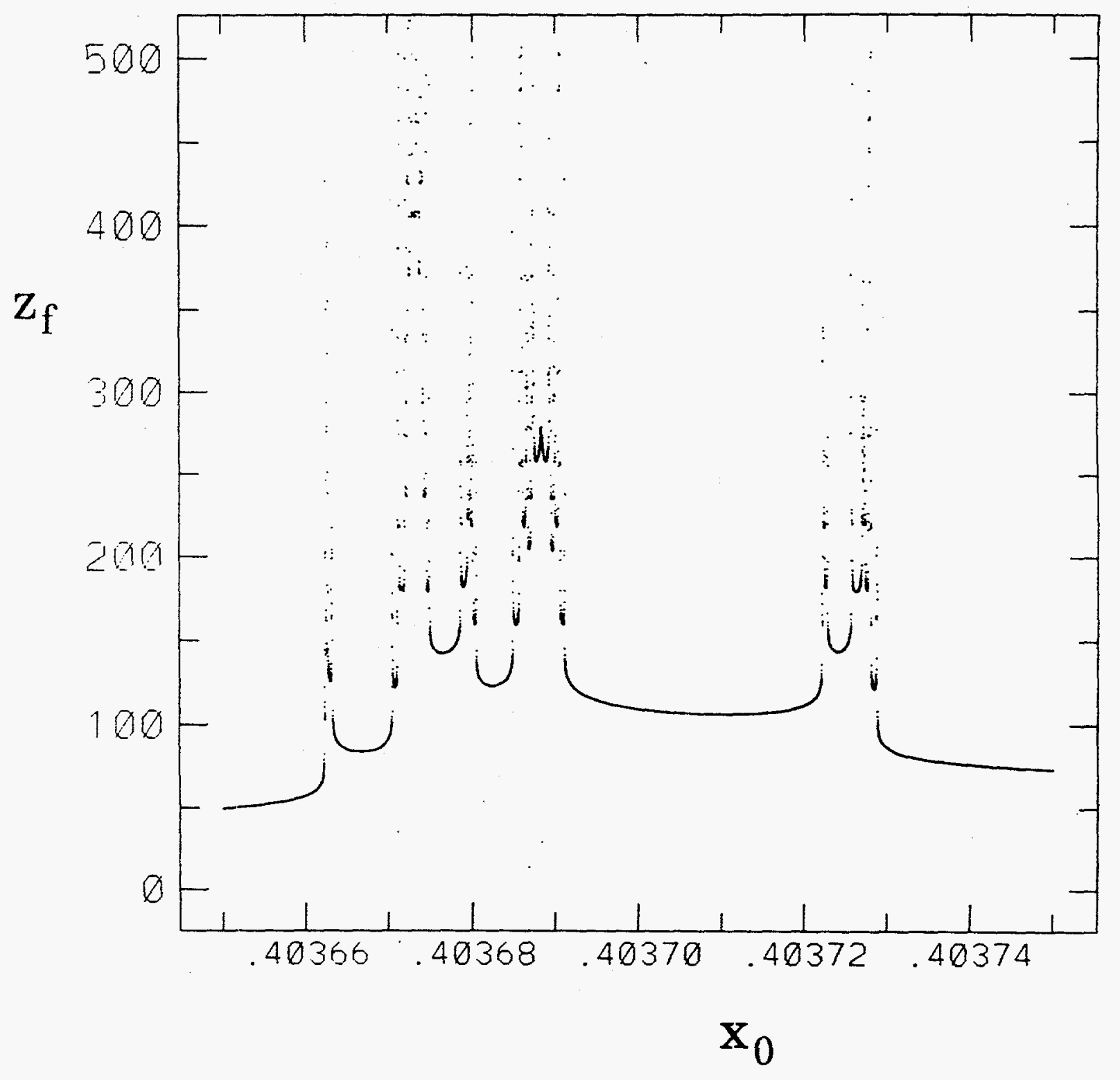

Fig. 9 
95PH044

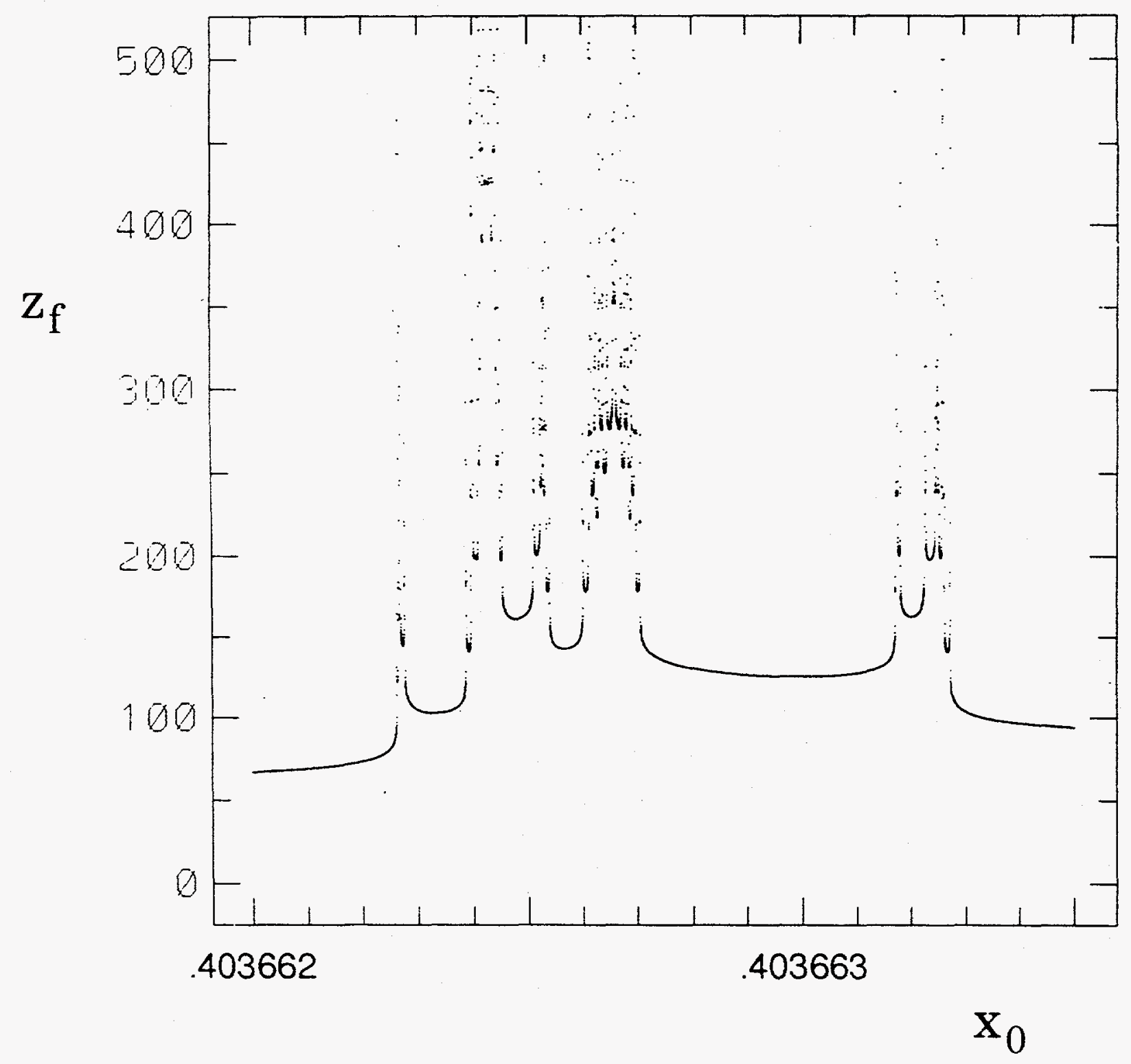

Fig. 10 
95PH045

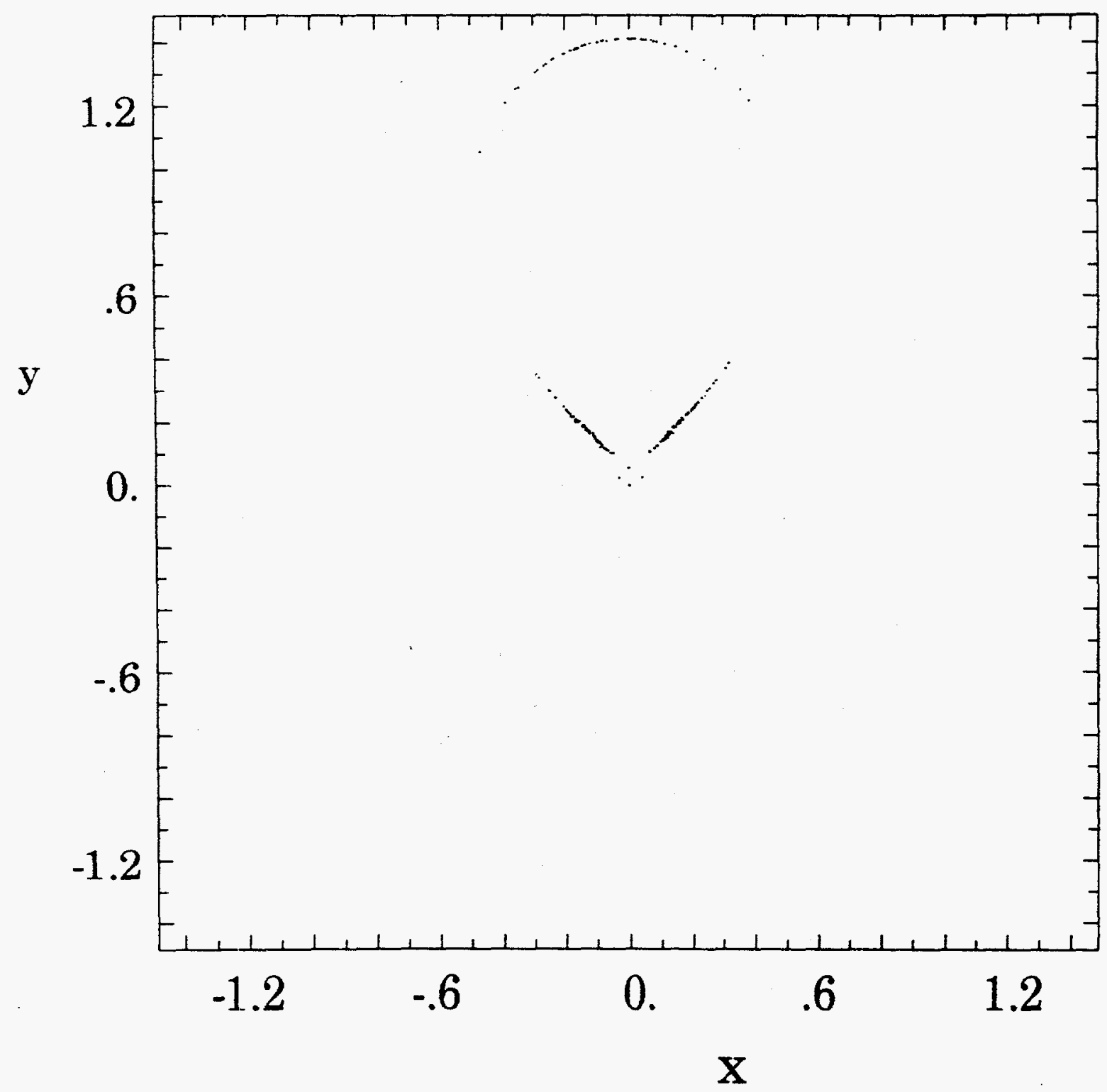

Fig. 11 
95PH046

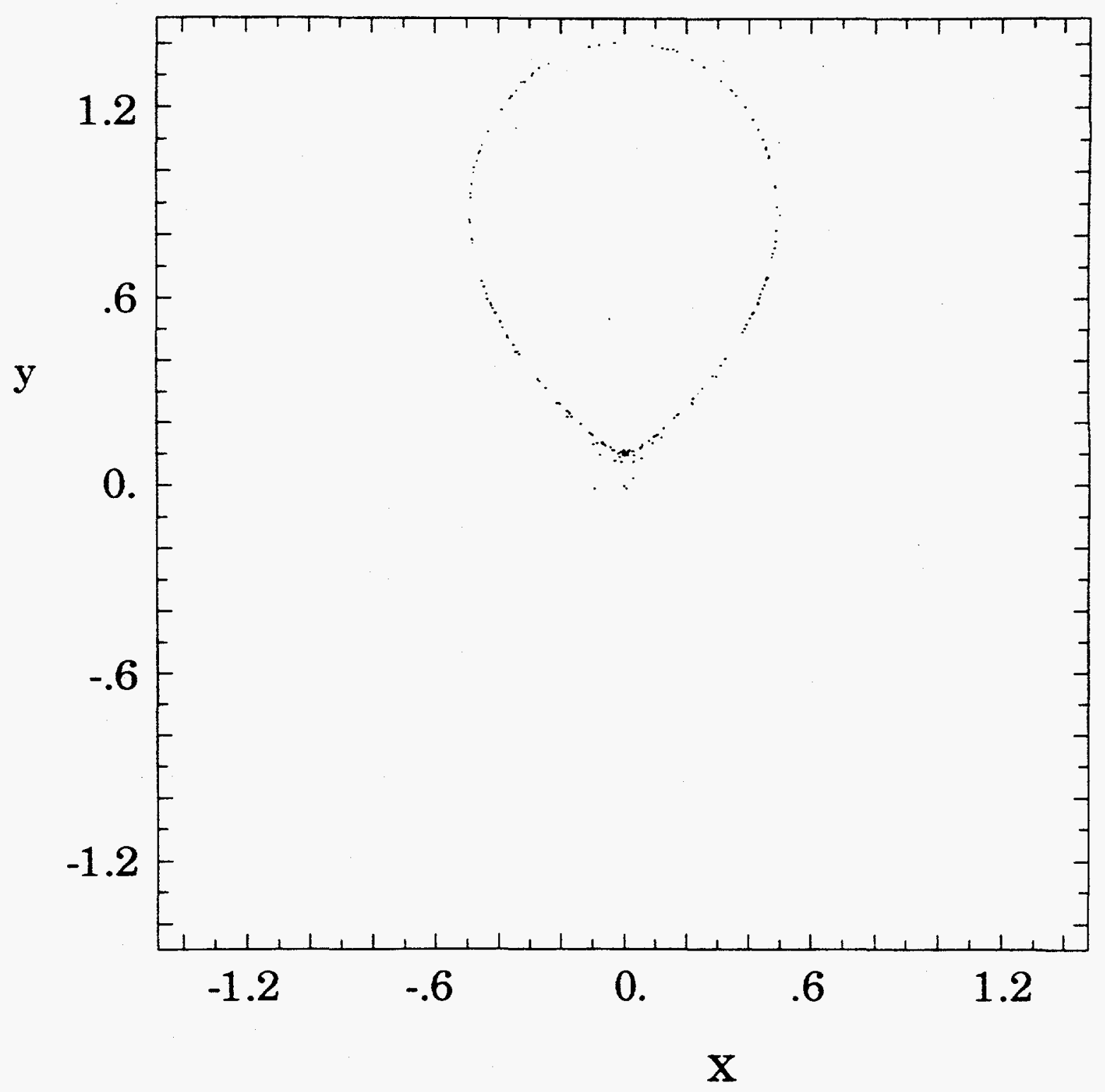

Eig. 12 
Dr. F. Paobni, Univ. of Wollongong, AUSTRALIA

Prof. R.C. Cross, Univ. of Sydney, AUSTRALIA

Plasma Research Lab., Australian Nat. Univ., AUSTRALIA

Prof. I.R. Jones, Flinders Univ, AUSTRALIA

Prof. F. Cap, Inst for Theoretical Physics, AUSTRIA

Prof. M. Heindler, Institut für Theoretische Physik, AUSTRIA

Prof. M. Goossens, Astronomisch Instituut, BELGIUM .

Ecole Royale Militaire, Lab. de Phy. Plasmas, BELGIUM

Commission-European, DG. XII-Fusion Prog., BELGIUM

Prof. R. Boucique, Rijksuniversiteit Gent, BELGIUM

Dr. P.H. Sakanaka, Instituto Fisica, BRAZIL

Prof. Dr. I.C. Nascimento, Instituto Fisica, Sao Paulo, BRAZIL Instituto Nacional De Pesquisas Espaciais-INPE, BRAZIL

Documents Office, Atomic Energy of Canada Ltd., CANADA

Ms. M. Morin, CCFWTokamak de Varennes, CANADA

Dr. M.P. Bactynski, MP8 Technologies, Inc., CANADA

Dr. H.M. Skarsgard, Univ. of Saskatchewan, CANADA

Prof. J. Teictmann, Univ. of Montreal, CANADA

Prof. S.R. Sreenivasan, Univ. of Calgary, CANADA

Prof. R. Marchand, INRS-Energie et Materiaux, CANADA

Dr. R. Bolton, Centre canadien de fusion magnétique, CANADA

Dr. C.R. James, Univ. of Alberta, CANADA

Dr. P. Lukác, Komenského Universzita, CZECHO-SLOVAKIA

The Librarian, Cutham Laboratory, ENGLAND

Library, R6:, Rutherford Appleton Laboratory, ENGLAND

Mrs. S.A. Hutchinson, JET Library, ENGLAND

Dr. S.C. Sharma, Univ. of South Pacific, FIJI ISLANDS

P. Mähönen, Univ. of Helsinki, FINLAND

Prof. M.N. Bussac, Ecole Polytechnique, FRANCE

C. Mouttet, Lab. de Physique des Milieux lonisés, FRANCE

J. Radet, CEN/CADARACHE - Bat 506, FRANCE

Prof. E. Economou, Univ. of Crete, GREECE

Ms. C. Rinni, Univ. of loannina, GREECE

Preprint Library, Hungarian Academy of Sai., HUNGARY

Dr. B. DasGupta, Saha Inst. of Nuclear Physics, INDIA

Dr. P. Kaw, Inst. for Plasma Research, INDIA.

Dr. P. Rosenau; Israel Inst. of Technology, ISRAEL

Librarian, Intemational Center for Theo Physics, ITALY

Miss C. De Paio, Associazione EURATOM-ENEA, ITALY

Dr. G. Grosso, Istituto di Fisica del Plasma, ITALY

Prof. G. Rostangni, Istituto Gas Ionizzati Del Cnr, ITALY
Dr. H. Yamato, Toshiba Res \& Devel Center, JAPAN

Prof. 1. Kawakami, Hiroshima Univ., JAPAN

Prof. K. Nishikawa, Hiroshima Univ., JAPAN

Librarian, Naka Fusion Research Establishment, JAERI, JAPAN

Director, Japan Atomic Energy Research Inst., JAPAN

Prof. S. Itoh, Kyushu Univ., JAPAN

Research info. Ctr., National Instit. for Fusion Scienć, JAPAN

Prof. S. Tanaka, Kyoto Univ., JAPAN

Library, Kyoto Univ., JAPAN

Prof. N. Inove, Univ. of Tokyo, JAPAN

Secretary, Plasma Section, Electrotechnical Lab., JAPAN

Dr. O. Mitarai, Kumamoto Inst. of Technology, JAPAN

Dr. G.S. Lee, Korea Basic Sci. Ctr., KOREA

J. Hyeon-Sook, Korea Atomic Energy Research Inst, KOREA

D.I. Choi, The Korea Adv. Inst. of Sci. \& Tect., KOREA

Leandro Melendez Lugo, Inst. Nac1. de Inves. Nud, MEXICO

Prof. B.S. Liley, Univ. of Waikato, NEW ZEALAND

Inst of Physics, Chinese Acad Sci PEOPLE'S REP. OF CHINA

Library, Inst. of Plasma Physics, PEOPLE'S REP. OF CHINA

Tsinghua Univ. Library, PEOPLE'S REPUBLIC OF CHINA

Z. Li, S.W. Inst Physics, PEOPLE'S REPUBLIC OF CHINA

Prof. J.A.C. Cabral, Instituto Superior Tecnico, PORTUGAL

Prof. M.A. Hellberg, Univ. of Natal, S. AFRICA

Prof. D.E. Kim, Pohang Inst. of Sci. \& Tech., SO. KOREA

Prof. C.I.E.M.A.T, Fusion Division Library, SPAIN

Dr. L Stenflo, Univ. of UMEA, SWEDEN

Library, Royal Inst. of Technology, SWEDEN

Prof. H. Wilheimson, Chalmers Univ. of Tech., SWEDEN

Centre Phys. Des Plasmas, Ecole Polytech, SWITZERLAND

Bibliotheek, Inst. Voor Plasma-Fysica, THE NETHERLANDS

Asst. Prof. Dr. S. Cakir, Middle East Tech. Univ., TURKEY

Dr. V.A. Glukhikh,Sci. Res. Inst. Electrophys.l Apparatus, USSR

Dr. D.D. Ryutov, Siberian Branch of Academy of Sci, USSR

Dr. G.A. Eliseev, I.V. Kurchatov Inst, USSR

Librarian, The Ukr.SSR Academy of Sciences, USSR

Dr. L.M Kovrizhnykh, Inst. of General Physics, USSR

Kemiorschungsanlage GmbH, Zentralbibliothek, W. GERMANY

Bibliothek, Inst. Für Plasmaforschung, W. GERMANY

Prof. K. Schindler, Ruhr-Universitát Bochum, W. GERMANY

Dr. F. Wagner, (ASDEX), Max-Planck-Institut, W. GERMANY

Librarian, Max-Planck-Institut, W. GERMANY 